\title{
Exercise training increased gene expression of LDL-R and PCSK9 in intestine: link to transintestinal cholesterol excretion
}

\author{
Zahra Farahnak ${ }^{1}$, Natalie Chapados ${ }^{2,3}$ and Jean-Marc Lavoie ${ }^{1}$ \\ ${ }^{1}$ Department of Kinesiology, University of Montreal, Montreal, QC, Canada \\ ${ }^{2}$ Research Institute of Montfort Hospital, Montfort Institute of Knowledge, ON, Canada \\ ${ }^{3}$ School of Human Kinetics, Faculty of Health Sciences, University of Ottawa, Ottawa, ON, Canada
}

\begin{abstract}
Transintestinal cholesterol excretion (TICE) is known as an alternate non-biliary route of cholesterol excretion from the body. The aim of this study was to determine whether exercise training has effects on intestinal membrane receptors involved in TICE in intact and ovariectomized (Ovx) rats. Sprague-Dawley rats were first divided into 4 groups: Sham operated and Ovx rats fed a standard diet (Sham-SD; Ovx-SD), or a high cholesterol diet (Sham-Chol; Ovx-Chol). These 4 groups were subsequently subdivided into either sedentary or voluntary wheel running groups for 6 weeks. The cholesterol diet resulted in increased hepatic cholesterol accumulation $(p<0.001)$ in both Sham and Ovx rats. Exercise training increased $(p<0.01)$ transcripts of intestinal low density lipoprotein receptor $(L D L-R)$ and proprotein convertase subtilisin/kexin type 9 (PCSK9), which are involved in trans-intestinal cholesterol uptake from circulation, in both Sham and Ovx rats compared to rats remaining sedentary in all diet conditions. The up-regulation of intestinal gene expression of $L D L-R$ and $P C S K 9$ following voluntary wheel running in intact and Ovx rats suggests that exercise training may contribute to elimination of cholesterol through the TICE pathway.
\end{abstract}

Key words: Intestinal cholesterol disposal — LDL-receptor — Liver cholesterol

\section{Introduction}

Although the biliary route is the main pathway for elimination of excess cholesterol from the body, several studies have recently enlighten that reverse cholesterol transport (RCT) can also proceed through a non-biliary pathway known as transintestinal cholesterol excretion (TICE) (Brown et al. 2008; van der Velde et al. 2008; Temel and Brown 2012). Indeed, the cholesterol disposal from the body depends on an active interplay between liver and intestine (Temel and Brown 2015). In TICE pathway, cholesterol is transported through the receptors at the basolateral and apical membrane

Electronic supplementary material. The online version of this article (doi: 10.4149/gpb_2017048) contains supplementary material which is available to authorized users.

Correspondence to: Zahra Farahnak, Department of Kinesiology, University of Montreal, CP 6128 succ « Centre-ville », Montréal, H3C3J7, Québec, Canada

E-mail: zahra.farahnak@umontreal.ca of intestine which are involved in cholesterol uptake from the circulation and cholesterol secretion into the lumen, respectively. Low density lipoprotein $(L D L)$ receptor and LDL receptor family were reported as receptors involved in cholesterol uptake from circulation at the basolateral side of intestine (Le May et al. 2013). Adenosine triphosphate binding cassette transporters G5 and G8 (ABCG5/G8) and adenosine triphosphate binding cassette transporters $\mathrm{B} 1 \mathrm{a}$ and $\mathrm{b}(A B C B 1 a / b)$ are introduced as receptors involved in cholesterol excretion into the lumen at the apical membrane of intestine (van der Veen et al. 2009; Le May et al. 2013). Transport and elimination of cholesterol is particularly relevant to ovariectomized (Ovx) animals in which hepatic cholesterol metabolism has been reported to be disrupted especially when fed the high cholesterol diet (Kamada et al. 2011; Cote et al. 2013). For instance, large hepatic cholesterol content was observed in Ovx rats fed a cholesterol diet (Subramanian et al. 2011; Cote et al. 2014). Recent studies have shown that hepatic mRNA expression of ABCG8 and bile salt export pump (BSEP) transcript, involved in cholesterol and bile acid excretion from liver into bile duct respectively, 
were also decreased in Ovx rats fed a cholesterol diet (Cote et al. 2014; Farahnak et al. 2015). It seems that estrogen withdrawal and high cholesterol diet act synergistically to impair different aspects of hepatic cholesterol metabolism including cholesterol excretion from the body. Under normal physiological conditions, the biliary route has a predominant role in cholesterol excretion and the non-biliary TICE pathway contributes to approximately $20-30 \%$ of the cholesterol disposal. However it has been reported that TICE pathway can be stimulated by both pathophysiological and pharmacological stimuli (Temel and Brown 2012). It seems that biliary cholesterol insufficiency can be compensated by intestine through TICE to keep normal levels of fecal cholesterol loss. Considering that hepatic cholesterol excretion is impaired in Ovx animal, thus it is important to gain knowledge about the role of TICE in cholesterol excretion in Ovx model.

There is some evidence that exercise training, as one of the best nonpharmacological strategies, attenuates hepatic cholesterol accumulation and leads to higher biliary bile acid secretion in hypercholesterolemic mice (Meissner et al. 2011). Increase in cholesterol 7 alpha-hydroxylase (CYP7A1) transcript, involved in conversion of cholesterol into bile acid in liver, was also reported in trained male mice (Pinto et al. 2015). Lack of evidence for training effects on TICE thus creates a great avenue to explore this pathway under training to gain a better knowledge of cholesterol disposal through intestine.

The aim of the present study was to determine the effect of exercise training on key intestinal cholesterol receptors involved in TICE in intact and Ovx rats fed a normal and a high cholesterol diet. We targeted gene expression of key molecules of TICE at intestinal basolateral membrane such as $L D L-R$ and proprotein convertase subtilisin/kexin type 9 (PCSK9) and also at intestinal apical membrane like ABCG5/ $G 8$ and $A B C B 1 a / b$. We also targeted gene expression of $L D L-R$, PCSK9 and scavenger receptor B1 (SR-B1) in liver.

\section{Materials and Methods}

\section{Animal care}

Eight week old female Sprague-Dawley strain rats $(n=65$; Charles River, St Constant, PQ, Canada), weighing 187$194 \mathrm{~g}$ upon their arrival were housed individually and had ad libitum access to food and tap water. Their environment was controlled in terms of light ( $12 \mathrm{~h}$ light-dark cycle starting at 06:00 AM), humidity and room temperature $\left(20-23^{\circ} \mathrm{C}\right)$. Body weight and food intake were monitored bi-weekly from the start of experiment. All experimental procedures were conducted according to the protocols approved by the Institutional Animal Care and Use Committee of the University of Montreal in agreement with The Canadian Council on Animal Care's rules (CCAC-CCPA).
Surgery, diets, and exercise protocol

Rats were first acclimated to their environment for a period of one week while fed a chow diet (12.5\% lipid, 63.2\% CHO and $24.3 \%$ protein; kJ from Agribrands Canada, Woodstock, Ontario, Canada). Afterwards, rats underwent either a bilateral ovariectomy (Ovx, $n=32$ ) or a bilateral sham-operation (Sham, $n=33$ ) according to the technique described by Robertson et al. (1984) under isoflurane anaesthesia. After surgery, all animals were injected with antibiotics (Tribrissen $48 \% ; 0.125 \mathrm{cc} / \mathrm{kg}$, subcutaneously) and analgesic (Carprofen; $4.4 \mathrm{mg} / \mathrm{kg}$, subcutaneously) for 3 days. Ovx and Sham rats were given either a standard diet (SD) or a high cholesterol diet (Chol). The Chol diet consisted of SD supplemented with $0.25 \%$ cholesterol (SD+Chol) (Table S1). The four groups composed of Sham rats fed a SD or a Chol diet (Sham-SD, $n=17$; Sham-Chol, $n=16$ and Ovx rats fed a SD or a Chol diet (Ovx-SD, $n=16$; Ovx-Chol, $n=16$ ) were further subdivided into either voluntary wheel running $(\mathrm{Tr})$ or sedentary groups (Sed) for a total of 8 groups. Tr rats were placed in freely rotating wheel cages while Sed rats were placed in blocked running wheel cages. Each wheel cage was equipped with a sensor connected to a computerized data acquisition system enabling the continuous sampling of running data from individual rats. Rats were on diet and training for 6 weeks.

\section{Blood and tissue sampling}

Rats were fasted overnight and euthanized between 08:00 and 11:00 AM. Rats were refrained from exercising $\sim 24 \mathrm{~h}$ before sacrifice. Immediately after complete anaesthesia with isoflurane, the abdominal cavity was opened following the median line of the abdomen. Approximately $5 \mathrm{ml}$ of blood was collected from the abdominal vena cava $(<45 \mathrm{~s})$ into syringes treated with ethylenediaminetetraacetic acid (15\%; EDTA). Blood was centrifuged $\left(3000 \mathrm{rpm} ; 4^{\circ} \mathrm{C} ; 10 \mathrm{~min}\right.$; Beckman GPR Centrifuge; Beckman Coulter) and the plasma kept for further analyses. Immediately after blood collection, the liver median lobe was removed and freeze-clamped. This sample was used for cholesterol, and mRNA determinations. Several organs were removed and weighed (with Analytical Balance Mettler AE 100) in the following order: uterus, urogenital, retroperitoneal and mesenteric fat deposits. The urogenital fat pad included adipose tissue surrounding the kidneys, uterus and bladder as well as ovaries, oviducts and uterus. The retroperitoneal fat pad was taken as that distinct deposit behind each kidney along the lumbar muscles. The mesenteric fat pad consisted of adipose tissue surrounding the gastrointestinal tract from the gastroesophageal sphincter to the end of the rectum, with special care taken in distinguishing and removing pancreatic cells. After mesenteric fat removal, a section of approximately $5 \mathrm{~cm}$ of jejunum, was removed, 
washed in antiprotease solution (10\% pepstatin A in methanol, 100\% leupeptin, $1.7 \%$ phenylmethylsulfonyl fluoride in isopropanol, $0.9 \% \mathrm{NaCl}$ ), and frozen in liquid nitrogen. Liver and a fragment of jejunum along with plasma samples were stored at $-78^{\circ} \mathrm{C}$ until analyses were performed.

\section{Biochemical analyses}

Liver total cholesterol concentrations were determined with some adaptations of the procedure described by Folch et al. (1957). Briefly, $0.1 \mathrm{~g}$ of liver was homogenized in a chloroform-methanol mixture $(2: 1, \mathrm{v} / \mathrm{v})$. The chloroform layer was collected and evaporated overnight. After adding $10 \%$ Triton X-100 in isopropanol, the sample was assayed for total cholesterol using commercial kits according to the manufacturer's instructions (Wako Diagnostics and Chemicals USA, Richmond, VA, USA). Plasma total cholesterol was determined using the same kit supplied by Wako. Plasma PCSK9 concentration was measured using Mouse/ Rat PCSK9 ELISA kit from CircuLex.

RNA isolation and quantitative real-time (RT) polymerase chain reaction ( $p C R$ )

Quick-frozen tissue samples of liver and jejunum were powdered with cold mortar and pestle and $\sim 100 \mathrm{mg}$ was used for the isolation of RNA. Details of RNA extraction following by RT-PCR have been previously described (Farahnak et al. 2015). The primer sets and UPL probe numbers used to generate amplicons are presented in Table S2.

\section{Statistical analysis}

All data are presented as mean \pm SE. Statistical significance $(p<0.05)$ was determined using a 3-way ANOVA for nonrepeated measures with exercise, diet and surgery as main factors. Fisher LSD post hoc test was used in the event of a significant interaction effect. For a significant exercise, diet and surgery effect without interaction, Fisher LSD from a one-way ANOVA was used.

\section{Results}

Anthropometric parameters, food intake and total distance run

Final body weight was not affected by exercise training in any of the experimental groups whereas intra-abdominal fat pad weight was significantly decreased $(p<0.001)$ by training in both Sham and Ovx rats regardless of type of the diet (Table $1)$. On the other hand, final body weight $(p<0.001)$, intraabdominal fat pad $(p<0.01)$ and also food intake $(p<0.001)$ were higher in Ovx compared to Sham rats implying the effect of estrogen withdrawal. Cholesterol diet had no impact on any of the aforementioned parameters in both Sham and Ovx rats. Uterus weight was significantly $(p<0.001)$ lower in Ovx groups compared to Sham rats confirming total ovariectomy (Table 1). Exercising rats ran on average $401 \pm$ $54 \mathrm{~min} /$ day thereby covering a total running distance of 8.9 $\pm 0.8 \mathrm{~km} /$ day (average speed: $1.36 \pm 0.18 \mathrm{~km} / \mathrm{h}$ ) and $9.1 \pm$ $0.8 \mathrm{~km} /$ day (average speed: $1.38 \pm 0.17 \mathrm{~km} / \mathrm{h}$ ) in Sham-SD and Sham-Chol rats respectively. Total running distance was $5.3 \pm 0.7 \mathrm{~km} /$ day (average speed: $0.79 \pm 0.1 \mathrm{~km} / \mathrm{h}$ ) and 4.4 $\pm 0.5 \mathrm{~km} /$ day (average speed: $0.68 \pm 0.18 \mathrm{~km} / \mathrm{h}$ ) in Ovx-SD and Ovx-Chol rats respectively.

\section{Molecular markers of TICE at the intestinal basolateral and apical membranes}

Running resulted in significantly $(p<0.01)$ higher $L D L-R$ transcript in both Sham and Ovx rats compared to rats remaining Sed in all diet conditions (Fig. 1). A trend for higher $L D L-R$ gene expression $(p=0.1)$ was observed under cholesterol feeding in both Sham and Ovx rats compared to

Table 1. Anthropometric parameters, food intake

\begin{tabular}{|c|c|c|c|c|c|c|c|c|}
\hline \multirow{2}{*}{ Variables } & \multicolumn{2}{|r|}{ Sham-SD } & \multicolumn{2}{|r|}{ Ovx-SD } & \multicolumn{2}{|c|}{ Sham-Chol } & \multicolumn{2}{|c|}{ Ovx-Chol } \\
\hline & Sed & $\operatorname{Tr}$ & Sed & $\operatorname{Tr}$ & Sed & $\operatorname{Tr}$ & Sed & $\operatorname{Tr}$ \\
\hline $\begin{array}{l}\text { Final body } \\
\text { weight (g) }\end{array}$ & $344.6 \pm 14.1$ & $317.4 \pm 11.4$ & $408.4 \pm 18.2^{\delta \delta \delta}$ & $378.5 \pm 24.9^{\delta \delta \delta}$ & $332.5 \pm 15.9$ & $314.4 \pm 17.4$ & $429.8 \pm 14.1^{\delta \delta \delta}$ & $424.9 \pm 18.5^{\delta \delta \delta}$ \\
\hline $\begin{array}{l}\text { Intra-abdominal } \\
\text { fat pad weights }(\mathrm{g})\end{array}$ & $28.9 \pm 4.7$ & $15.1 \pm 4^{\star \star \star}$ & $34.7 \pm 5^{\delta \delta}$ & $26.2 \pm 5.8^{\star * \star}, \delta \delta$ & $29.8 \pm 4.5$ & $14.1 \pm 3.7^{\star \star *}$ & $39.2 \pm 3.5^{\delta \delta}$ & $30.1 \pm 4.9^{* * *, \delta \delta}$ \\
\hline $\begin{array}{l}\text { Food intake } \\
\text { (kcal/day) }\end{array}$ & $83.2 \pm 3.4$ & $86.1 \pm 3.3$ & $92.9 \pm 5.7^{\delta \delta \delta}$ & $95.6 \pm 5.6^{\delta \delta \delta}$ & $81.6 \pm 4.1$ & $89.4 \pm 3.1$ & $99.2 \pm 3.9^{\delta \delta \delta}$ & $104.9 \pm 5.7^{\delta \delta \delta}$ \\
\hline Uterus (g) & $0.45 \pm 0.07$ & $0.41 \pm 0.1$ & $0.11 \pm 0.02^{\delta \delta \delta}$ & $0.12 \pm 0.03^{\delta \delta \delta}$ & $0.41 \pm 0.04$ & $0.48 \pm 0.05$ & $0.12 \pm 0.02^{\delta \delta \delta}$ & $0.11 \pm 0.04^{\delta \delta \delta}$ \\
\hline
\end{tabular}

Ovx, ovariectomised; Sham, sham operated; SD, standard diet; Chol, standard diet $+0.25 \%$ cholesterol; Sed, sedentary group; Tr, trained group. Values are mean \pm SE. Significantly different from respective Sed group: ${ }^{* *} p<0.001$; significantly different from respective Sham rats: ${ }^{\delta} p<0.01 ;{ }^{\delta} \delta \delta<0.001$. 

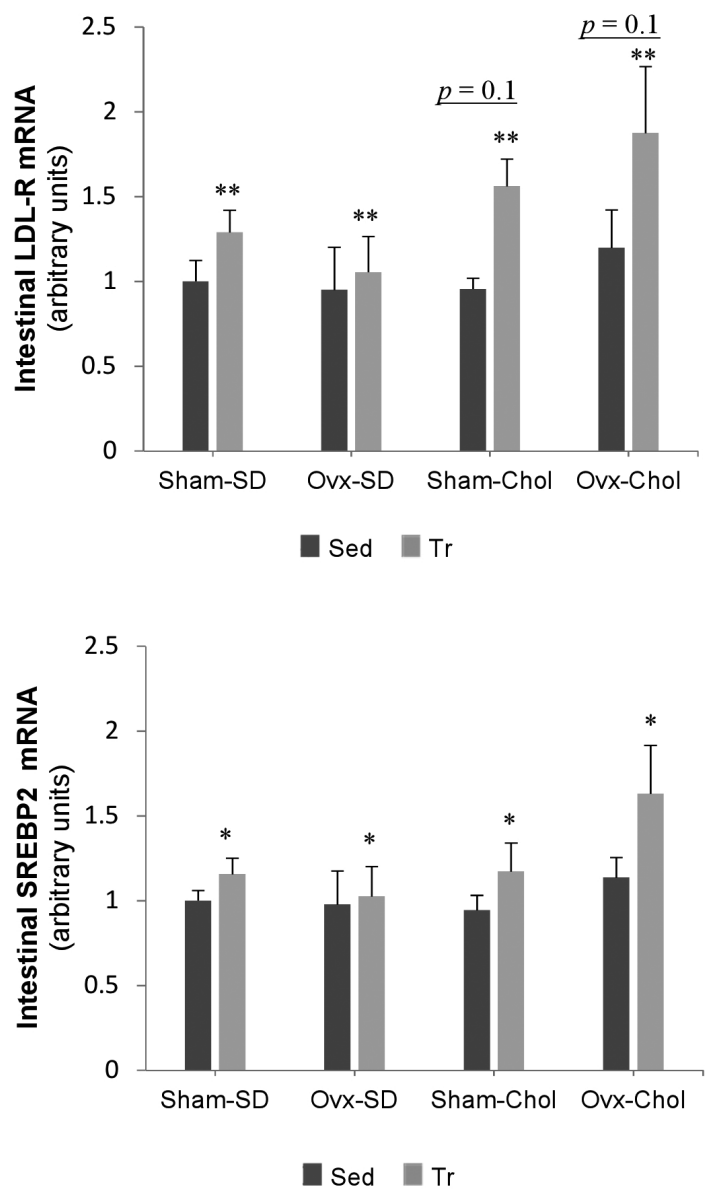

rats on the SD diet. Similar to $L D L-R, P C S K 9$ mRNA gene expression was significantly $(p<0.001)$ higher following training but only in rats fed the Chol diet. These two genes are involved in cholesterol uptake from circulation at the basolateral side of intestine. $L D L-R$ and $P C S K 9$ are the target genes of transcription factor $S R E B P 2$. Intestinal SREBP2 transcript was also increased $(p<0.05)$ by training in both Sham and Ovx rats in both dietary conditions (Fig. 1). On the other hand, estrogen withdrawal had no effect on $L D L-R$ and PCSK9 mRNA gene expression. In addition to $L D L-R$ and PCSK9, scavenger receptor class B member 1 (SR-B1) and very low density lipoprotein receptor $(V L D L-R)$ may also play a role in intestinal cholesterol uptake from plasma. Our results showed that mRNA expression of $S R-B 1$ and $V L D L-$ $R$ were not affected by training, Chol diet, and or estrogen withdrawal in any of the experimental groups (Fig. S1).

ATP-binding cassette protein G5/G8 (ABCG5/G8) and ATP-binding cassette sub-family B member $1 \mathrm{a} / \mathrm{b}$ $(A B C B 1 a / b)$, the receptors at the apical membrane of the intestine, are involved in cholesterol excretion from the intestine into the lumen in TICE pathway. With the exception of ABCB1b mRNA expression which was found to be lower

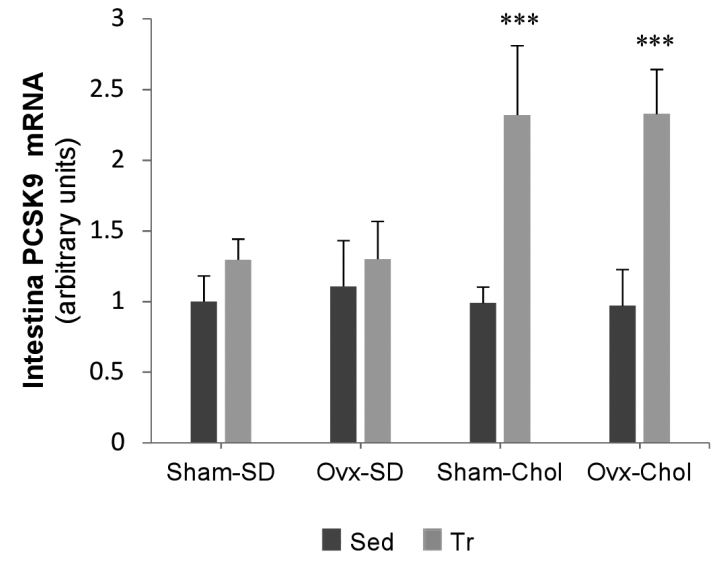

Figure 1. mRNA expression of genes involved in transintestinal cholesterol excretion (TICE) at the basolateral membrane of the intestine in sham operated rats fed a standard diet (Sham-SD), ovariectomized rats fed a standard diet (Ovx-SD), Sham rats fed a standard diet $+0.25 \%$ cholesterol (Sham-Chol) and Ovx rats fed the Chol diet (Ovx-Chol) in sedentary (Sed) or trained (Tr) state. Values are mean \pm SE. Significantly different from respective Sed rats: ${ }^{\star} p<0.05,{ }^{* *} p<0.01,{ }^{* * *} p<0.001$. LDL-R, low density lipoprotein receptor; PCSK9, proprotein convertase subtilisin/ kexin type 9; SREBP2, sterol regulatory element-binding protein 2.

$(p<0.05)$ in Sham compared to Ovx rats (Fig. 2), all other receptors measured at the intestinal apical side were not affected by any of the main experimental factors (exercise, diet, surgery) (Fig. 2).

\section{Liver and plasma total cholesterol (TC) levels}

Running had no effect on liver and plasma TC levels (Fig. 3). Cholesterol diet resulted in higher $(p<0.001)$ hepatic TC in both Sham and Ovx rats compared to animals on SD diet. This result indicates that cholesterol feeding led to cholesterol accumulation in liver. Plasma TC levels was not affected by the dietary intervention (Fig. 3). Estrogen withdrawal had no impact on TC levels in liver while levels of TC in plasma were higher $(p<0.01)$ in Ovx compared to Sham rats fed the Chol diet.

\section{Molecular markers of hepatic cholesterol uptake form} circulation

Running had no impact on mRNA gene expression of hepatic $L D L-R, P C S K 9$ and circulating PCSK9 (Fig. 4) while SR-B1 
transcript, involved in cholesterol uptake from HDL, was higher $(p<0.05)$ in Tr than in Sed animals in both Sham and Ovx groups. Cholesterol feeding significantly reduced mRNA expression of all of these three genes in both Sham and Ovx groups compared to rats fed the SD diet with the exception of PCSK9 gene expression which was only decreased in Sham-Chol group (Fig. 4). Ovx rats showed lower $L D L-R$ transcript $(p<0.001)$ compared to Sham rats in both dietary conditions. On the other hand, PCSK9 mRNA expression was only decreased $(p<0.05)$ by estrogen withdrawal in Ovx rats on SD diet compared to Sham-SD group. SR-B1 mRNA expression was not affected by estrogen withdrawal in any of the experimental groups. Circulating concentration of PCSK9 showed the same trend as its hepatic gene expression (Fig. 4). Taken all together, cholesterol feeding and estrogen deficiency state had major effects on reduction of the expression of the receptors involved in cholesterol uptake from circulation in liver, especially in $L D L-R$ and PCSK9.

\section{Discussion}

The main finding of the present study was that a six week of voluntary exercise resulted in a significant increase in gene expression of intestinal $L D L-R$ and PCSK9 at the basolateral membrane along with their regulatory transcription factor SREBP2. This was observed especially under the Chol-fed condition independently of the Ovx or Sham surgery. Unlike the basolateral receptors, gene expressions of the intestinal apical receptors $(A B C G 5 / G 8$ and $A B C B 1 a / b)$ involved in cholesterol excretion from intestine into the lumen were not affected by exercise training. These results indicate that intestinal cholesterol uptake from circulation might be increased at the basolateral membrane of intestine following exercise training. This suggests that TICE route might be an explanation how exercise training contributes to an elimination of excess cholesterol.

To the best of our knowledge, the present study is the first to report an important increase in intestinal $L D L-R$
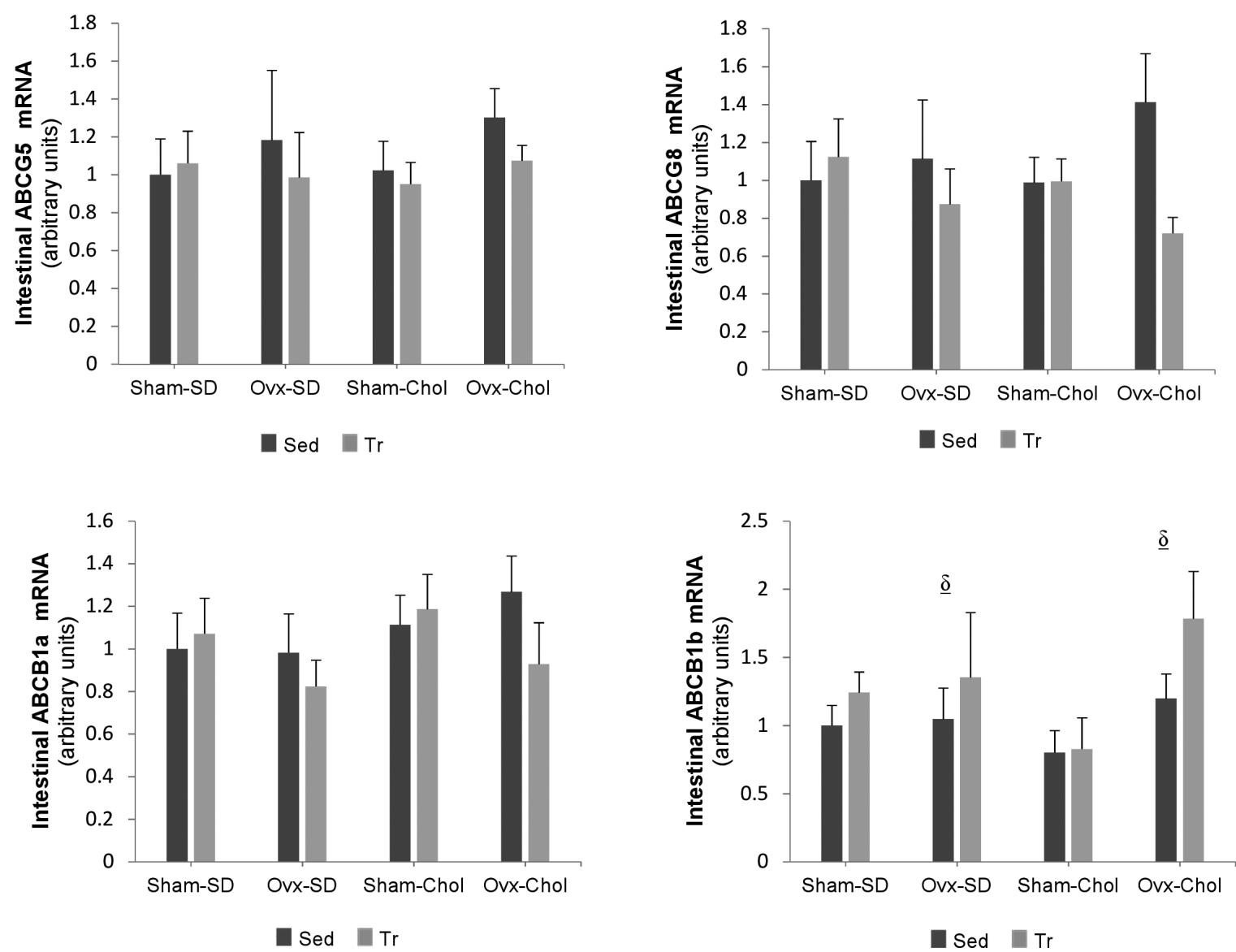

Figure 2. mRNA expression of genes involved in transintestinal cholesterol excretion (TICE) at the apical membrane of the intestine in sham operated rats fed a standard diet (Sham-SD), ovariectomized rats fed a standard diet (Ovx-SD), Sham rats fed a standard diet + $0.25 \%$ cholesterol (Sham-Chol) and Ovx rats fed the Chol diet (Ovx-Chol) in sedentary (Sed) or trained (Tr) state. Values are mean \pm SE. Significantly different from respective Sham rats ${ }^{\delta} p<0.05$. ABCG5, ATP-binding cassette protein G5; ABCG8, ATP-binding cassette protein G8; ABCB1a, ATP-binding cassette sub-family B member 1a; ABCB1b, ATP-binding cassette sub-family B member 1b. 

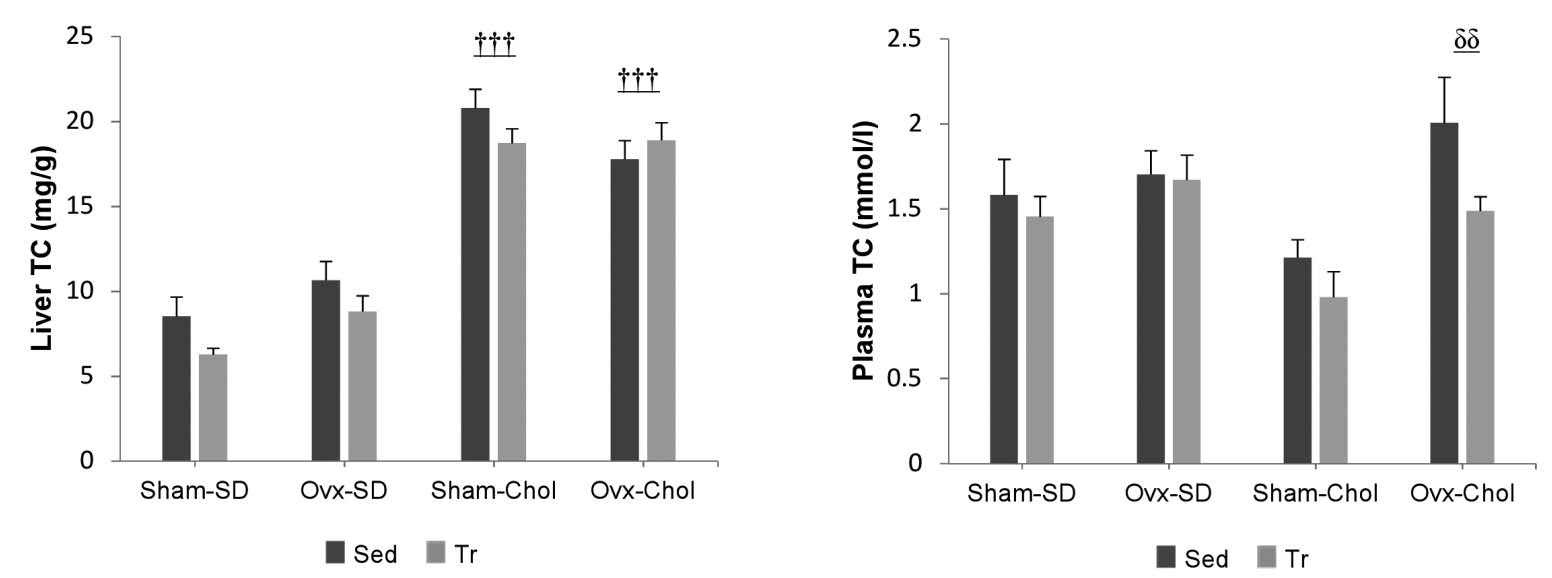

Figure 3. Liver and plasma total cholesterol (TC) levels in sham operated rats fed a standard diet (Sham-SD), ovariectomized rats fed a standard diet (Ovx-SD), Sham rats fed a standard diet $+0.25 \%$ cholesterol (Sham-Chol) and Ovx rats fed the Chol diet (Ovx-Chol) in sedentary (Sed) or trained (Tr) state. Values are mean \pm SE. Significantly different from respective rats fed the SD: ${ }^{\dagger \dagger \dagger} p<0.001$; significantly different from respective Sham rats: ${ }^{\delta \delta} p<0.01$.

and PCSK9 transcripts following exercise training. The main function of LDL-receptor is to remove the circulating cholesterol from apoB-lipoproteins (Ouguerram et al. 2004). LDLreceptor activity is downregulated post-transcriptionally by PCSK9 (Abifadel et al. 2003). Both $L D L-R$ and PCSK9 are regulated transcriptionally by the transcription factor, SREBP-2 (Smith et al. 1990; Dubuc et al. 2004). Although protein levels were not measured in the present study, there is consistency in the responses of intestinal LDL-R and PCSK9 transcripts and their nuclear transcription receptor, intestinal SREBP-2, in response to exercise training. Increased intestinal PCSK9 transcript along with LDL-R in trained rats might raise the possibility that there is degradation of intestinal $L D L-R$ through PCSK9. However, circulating concentration of PCSK9 was significantly low in Ovx rats and Chol-fed rats compared to intact rats on SD diet suggesting less degradation effect of PCSK9 on intestinal LDL-R. This might also explain why the plasma TC levels did not increase in Chol-fed rats in our study.

On the other hand, it has been reported that circulating recombinant PCSK9 injection through degradation of LDL-R content in intestine acutely decreases TICE in PCSK9 knockout mice (Schmidt et al. 2008; Le May et al. 2013). Moreover, it was reported that PCSK9 null mice have more LDL receptors in their gut (Le May et al. 2009) and subsequently faster intestinal cholesterol clearance and higher TICE were observed in PCSK9 null mice compared to wild type (WT) mice (Le May et al. 2013). On the other hand, injection of recombinant PCSK9 in LDL-R null mice resulted in 40\% higher TICE than PCSK9 knockout mice. The discrepancy between the effect of circulating PCSK9 on TICE were explained by the existence of higher TICE in LDL$\mathrm{R}^{-/-}$mice or the difference in genetic background between strains (Le May et al. 2013). Indeed, different levels of TICE were reported in mice with different genetic backgrounds (van der Velde et al. 2007).

Le May et al. (2013) recently suggested that in addition to LDL-R, other unidentified mechanisms might be involved in cholesterol uptake from plasma via TICE due to the uptake of LDL particles at the proximal part of intestine in LDL- $\mathrm{R}^{-/}$ mice. In addition to intestinal LDL-R, SR-B1 and VLDL-R have been identified as cholesterol acceptors at the basolateral membrane of intestine as well. Contrary to intestinal $L D L-R$, intestinal $S R-B 1$ and $V L D L-R$ transcripts were not altered following exercise training. SR-B1 has a well-accepted role in RCT via hepatobiliary pathway (Tall et al. 2008), It binds with apolipoprotein A-I (apoA-I), a protein component of highdensity lipoprotein (HDL), and removes esterified cholesterol from HDL (Acton et al. 1996). van der Velde et al. (2008) reported that upregulation of intestinal $S R-B 1$ expression by high-fat feeding was correlated with TICE. However, in the same study they surprisingly found that intestinal perfusions resulted in twofold increase in TICE in SR-B1 deficient compared with WT mice. It seems that despite the well-known role of $S R-B 1$ in hepatobiliary, its function in TICE route is unclear. In addition, Vrins CL, et al. reported that secretion of radiolabeled cholesterol from HDL via TICE did not change in $\mathrm{WT}$ and $\mathrm{ABCA} 1^{-/-}$and SR-1 ${ }^{-/-}$mice, implying that HDL might not be the plasma cholesterol donor to intestine (Vrins et al. 2012) and consequently it is reasonable to assume that $S R$-B1 might not have a significant role in cholesterol uptake from circulation at the intestinal basolateral side (Bura et al. 2013). Based on these findings and the result of the present study, it appears that LDL-R might be the main cholesterol acceptor from circulation at the basolateral side of intestine influenced by exercise training. 
Surprisingly, we found no effects of exercise training as well as Chol-diet and estrogen withdrawal on intestinal receptors (except $A B C B 1 b$ transcript) at the apical side which are involved in cholesterol excretion from intestine into the lumen. Intestinal $A B C B 1 b$ transcript was higher in Ovx rats and particularly showed a tendency to higher expression in Ovx trained rats however it did not reach the point to be significant. Previously, van der Veen et al. (2009) showed that TICE is impaired in Abcg 5 null mice, implying that intestinal cholesterol transporting ABCG5/ G8 heterodimer contributes to TICE pathway. On the other hand, it has been shown that mice lacking ABCG5 still have an appropriate level of TICE, suggesting that there are other apical transporters that have a role in TICE. Le May et al. (2013) showed that in addition to $A B C G 5 / G 8$, multidrug transporter $A B C B 1 a / b$, located at the apical side of enterocyte is also involved in intestinal cholesterol excretion into the lumen. On the whole further studies need to be done to clarify what receptors might have the main role in cholesterol excretion at the basolateral side of intestine. In regard to exercise training, there is inconsistency in previous reports on the expression of cholesterol transporters at the intestinal apical side. Some authors reported an increase in intestinal $A B C G 8$ gene and protein expression in treadmill-trained female rats compared to female Sed rats (Ghanbari-Niaki et al. 2012). On the other hand, reduced $A B C G 8$ gene expression was observed in the ileum of exercise-trained female rats compared to Sed female rat (Ngo Sock et al. 2014b) probably due to a reduced need to efflux cholesterol back to the lumen as a consequence of lower cholesterol absorption (Wilund et al. 2008). Apparently, exercise training could promote TICE through increase in gene expression of receptors involved in cholesterol uptake from circulation at the basolateral
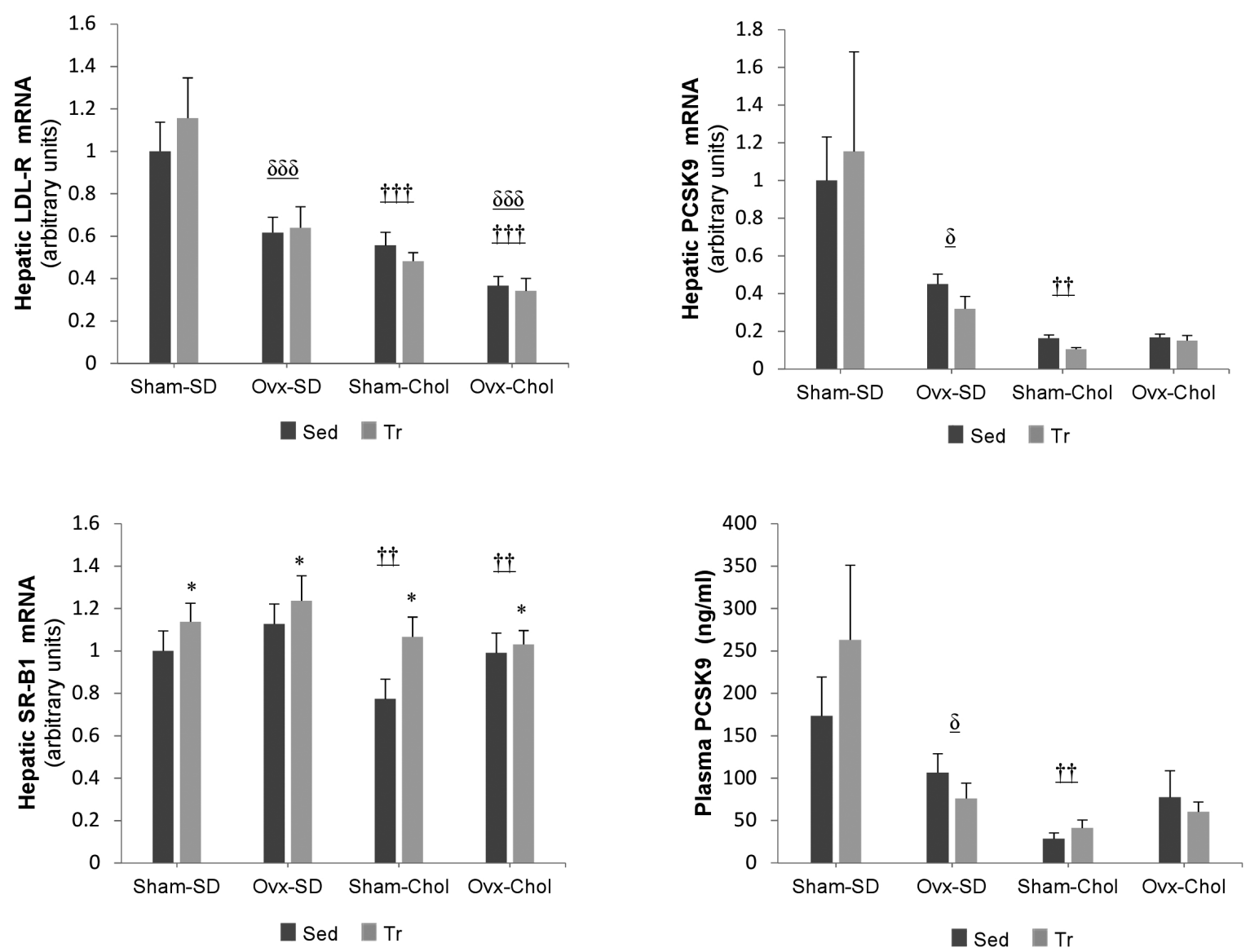

Figure 4. Hepatic mRNA expression of genes involved in cholesterol uptake from circulation and also plasma Pcsk9 levels in sham operated rats fed a standard diet (Sham-SD), ovariectomized rats fed a standard diet (Ovx-SD), Sham rats fed a standard diet $+0.25 \%$ cholesterol (Sham-Chol) and Ovx rats fed the Chol diet (Ovx-Chol) in sedentary (Sed) or trained (Tr) state. Values are mean \pm SE. Significantly different from respective Sed rats: ${ }^{*} p<0.05$; significantly different from respective rats fed the SD: ${ }^{\dagger \dagger} p<0.01,{ }^{\dagger \dagger} p<0.001$; significantly different from respective Sham rats: ${ }^{\delta} p<0.05,{ }^{\delta \delta \delta} p<0.001$. LDL-R, low density lipoprotein receptor; PCSK9, proprotein convertase subtilisin/kexin type 9; SR-B1, Scavenger receptor class B member 1. 
side, however further works will be needed to illustrate the excretion of cholesterol from intestinal apical side into the lumen.

In contrast to intestinal $L D L-R$ and PCSK9 transcripts, hepatic gene expression of $L D L-R$ and PCSK9 were not altered by exercise training. Hepatic $L D L-R$ transcript was, however, lowered by the ovariectomy and the Chol feeding. Unchanged hepatic transcripts of $L D L-R$ and PCSK9 following training in the present study was in concert with the recent observation that exercise training had no effects on hepatic gene expression of $L D L-R$ and PCSK9 in Ovx rats (Ngo Sock et al. 2014a). On the other hand, it was also reported that treadmill exercise resulted in increased LDL-R, PCSK9 and SREBP-2 mRNA expression in liver of high-fat-fed C57BL/6 mice. Reduction in hepatic cholesterol accumulation was mentioned as a main reason for higher hepatic $L D L-R, P C S K 9$ transcript by treadmill exercise in high-fat-fed C57BL/6 mice (Wen et al. 2013). It seems that higher hepatic cholesterol content in Chol-fed rats in our study is an underlying reason for lower hepatic $L D L-R$, PCSK9 transcript and consequently suppression of cholesterol uptake from plasma. In addition, higher expression of $S R-B 1$, involved in cholesterol uptake from circulating HDL, by training and simultaneously its down regulation following Chol-diet led us to the interpretation that this could probably be a protective response to prevent more cholesterol accumulation in the liver of Ovx rats. Taken all together, it appears that the effects of exercise training on the management of cholesterol metabolism may happen more at the intestinal than at the hepatic level in our animal model.

Considering that cholesterol uptake was reduced through the liver probably due to hepatic cholesterol accumulation, it was expected to observe higher plasma TC levels under cholesterol feeding. The absence of increased plasma TC levels in the present study might be explained by higher cholesterol uptake through intestinal LDL-R as a result of intestinal up-regulation of this receptor under training. The present finding that exercise training up-regulated the gene expression of intestinal $L D L-R$ and PCSK9 in the Ovx rats extends the previous findings from our lab showing that exercise training seemingly provokes estrogenic like effects on the expression of several genes for instance the genes involved in the regulation of lipid metabolism in liver (Pighon et al. 2011). However, the underlying mechanisms are still unknown and need future investigation.

In summary, results of the present study indicate that exercise training through up-regulation of the intestinal gene expression of $L D L-R$ and PCSK9 in intact and Ovx rats may contribute to elimination of excess cholesterol via TICE pathway. It also introduced exercise training as an appropriate non-pharmacological intervention to stimulate TICE to excrete the extra cholesterol from the body and decrease the risk of atherosclerosis.
Conflict of interest. The authors declare that they have no conflict of interest.

Acknowledgements. This work was supported by grants from the Natural Sciences and Engineering Research Council of Canada (NSERC; 7594).

\section{References}

Abifadel M, Varret M, Rabes JP, Allard D, Ouguerram K, Devillers M, Cruaud C, Benjannet S, Wickham L, Erlich D, et al. (2003): Mutations in PCSK9 cause autosomal dominant hypercholesterolemia. Nat. Genet. 34, 154-156 https://doi.org/10.1038/ng1161

Acton S, Rigotti A, Landschulz KT, Xu S, Hobbs HH, Krieger M (1996): Identification of scavenger receptor SR-BI as a high density lipoprotein receptor. Science 271, 518-520 https://doi.org/10.1126/science.271.5248.518

Brown JM, Bell TA, Alger HM, Sawyer JK, Smith TL, Kelley K, Shah R, Wilson MD, Davis MA, Lee RG, et al. (2008): Targeted depletion of hepatic ACAT2-driven cholesterol esterification reveals a non-biliary route for fecal neutral sterol loss. J. Biol. Chem. 283, 10522-10534 https://doi.org/10.1074/jbc.M707659200

Bura KS, Lord C, Marshall S, McDaniel A, Thomas G, Warrier M, Zhang J, Davis MA, Sawyer JK, Shah R, et al. (2013): Intestinal SR-BI does not impact cholesterol absorption or transintestinal cholesterol efflux in mice. J. Lipid Res. 54, 1567-1577 https://doi.org/10.1194/jlr.M034454

Cote I, Ngo Sock ET, Levy E, Lavoie JM (2013): An atherogenic diet decreases liver FXR gene expression and causes severe hepatic steatosis and hepatic cholesterol accumulation: effect of endurance training. Eur. J. Nutr. 52, 1523-1532 https://doi.org/10.1007/s00394-012-0459-5

Cote I, Chapados NA, Lavoie JM (2014): Impaired VLDL assembly: a novel mechanism contributing to hepatic lipid accumulation following ovariectomy and high-fat/high-cholesterol diets? Br. J. Nutr. 112, 1592-1600 https://doi.org/10.1017/S0007114514002517

Dubuc G, Chamberland A, Wassef H, Davignon J, Seidah NG, Bernier L, Prat A (2004): Statins upregulate PCSK9, the gene encoding the proprotein convertase neural apoptosis-regulated convertase-1 implicated in familial hypercholesterolemia. Arterioscler. Thromb. Vasc. Biol. 24, 1454-1459 https://doi.org/10.1161/01.ATV.0000134621.14315.43

Farahnak Z, Côté I, Sock ETN, Lavoie J-M (2015): High dietary cholesterol and ovariectomy in rats repress gene expression of key markers of VLDL and bile acid metabolism in liver. Lipids Health Dis. 14, 125-134 https://doi.org/10.1186/s12944-015-0128-9

Folch J, Lees M, Sloane-Stanley G (1957): A simple method for the isolation and purification of total lipids from animal tissues. J. Biol. Chem. 226, 497-509

Ghanbari-Niaki A, Rahmati-Ahmadabad S, Zare-Kookandeh N (2012): ABCG8 Gene responses to 8 weeks treadmill running with or without Pistachia atlantica (Baneh) extraction in female rats. Int. J. Endocrinol. Metab. 10, 604-610 
https://doi.org/10.5812/ijem.5305

Kamada Y, Kiso S, Yoshida Y, Chatani N, Kizu T, Hamano M, Tsubakio M, Takemura T, Ezaki H, Hayashi N, Takehara T (2011): Estrogen deficiency worsens steatohepatitis in mice fed high-fat and high-cholesterol diet. Am. J. Physiol. Gastrointest. Liver Physiol. 301, G1031-1043 https://doi.org/10.1152/ajpgi.00211.2011

Le May C, Kourimate S, Langhi C, Chetiveaux M, Jarry A, Comera C, Collet X, Kuipers F, Krempf M, Cariou B, Costet P (2009): Proprotein convertase subtilisin kexin type 9 null mice are protected from postprandial triglyceridemia. Arterioscler. Thromb. Vasc. Biol. 29, 684-690 https://doi.org/10.1161/ATVBAHA.108.181586

Le May C, Berger JM, Lespine A, Pillot B, Prieur X, Letessier E, Hussain MM, Collet X, Cariou B, Costet P (2013): Transintestinal cholesterol excretion is an active metabolic process modulated by PCSK9 and statin involving ABCB1. Arterioscler. Thromb. Vasc. Biol. 33, 1484-1493 https://doi.org/10.1161/ATVBAHA.112.300263

Meissner M, Lombardo E, Havinga R, Tietge UJ, Kuipers F, Groen AK (2011): Voluntary wheel running increases bile acid as well as cholesterol excretion and decreases atherosclerosis in hypercholesterolemic mice. Atherosclerosis 218, 323-329 https://doi.org/10.1016/j.atherosclerosis.2011.06.040

Ngo Sock ET, Chapados NA, Lavoie JM (2014a): LDL receptor and Pcsk9 transcripts are decreased in liver of ovariectomized rats: effects of exercise training. Horm. Metab. Res. 46, 550-555 https://doi.org/10.1055/s-0034-1370910

Ngo Sock ET, Farahnak Z, Lavoie JM (2014b): Exercise training decreases gene expression of endo- and xeno-sensors in rat small intestine. Appl. Physiol. Nutr. Metab. 39, 1098-1103 https://doi.org/10.1139/apnm-2013-0573

Ouguerram K, Chetiveaux M, Zair Y, Costet P, Abifadel M, Varret M, Boileau C, Magot T, Krempf M (2004): Apolipoprotein B100 metabolism in autosomal-dominant hypercholesterolemia related to mutations in PCSK9. Arterioscler. Thromb. Vasc. Biol. 24, 1448-1453 https://doi.org/10.1161/01.ATV.0000133684.77013.88

Pighon A, Gutkowska J, Jankowski M, Rabasa-Lhoret R, Lavoie JM (2011): Exercise training in ovariectomized rats stimulates estrogenic-like effects on expression of genes involved in lipid accumulation and subclinical inflammation in liver. Metabolism 60, 629-639 https://doi.org/10.1016/j.metabol.2010.06.012

Pinto PR, Rocco DD, Okuda LS, Machado-Lima A, Castilho G, da Silva KS, Gomes DJ, Pinto Rde S, Iborra RT, Ferreira Gda S, et al. (2015): Aerobic exercise training enhances the in vivo cholesterol trafficking from macrophages to the liver independently of changes in the expression of genes involved in lipid flux in macrophages and aorta. Lipids Health Dis. 14, 109-120 https://doi.org/10.1186/s12944-015-0093-3

Robertson MC, Owens RE, Klindt J, Friesen HG (1984): Ovariectomy leads to a rapid increase in rat placental lactogen secretion. Endocrinology 114, 1805-1811 https://doi.org/10.1210/endo-114-5-1805

Schmidt RJ, Beyer TP, Bensch WR, Qian YW, Lin A, Kowala M, Alborn WE, Konrad RJ, Cao G (2008): Secreted proprotein convertase subtilisin/kexin type 9 reduces both hepatic and extrahepatic low-density lipoprotein receptors in vivo. Biochem. Biophys. Res. Commun. 370, 634-640

https://doi.org/10.1016/j.bbrc.2008.04.004

Smith JR, Osborne TF, Goldstein JL, Brown MS (1990): Identification of nucleotides responsible for enhancer activity of sterol regulatory element in low density lipoprotein receptor gene. J. Biol. Chem. 265, 2306-2310

Subramanian S, Goodspeed L, Wang S, Kim J, Zeng L, Ioannou GN, Haigh WG, Yeh MM, Kowdley KV, O'Brien KD, Pennathur S, Chait A (2011): Dietary cholesterol exacerbates hepatic steatosis and inflammation in obese LDL receptor-deficient mice. J. Lipid Res. 52, 1626-1635

https://doi.org/10.1194/jlr.M016246

Tall AR, Yvan-Charvet L, Terasaka N, Pagler T, Wang N (2008): $\mathrm{HDL}, \mathrm{ABC}$ transporters, and cholesterol efflux: implications for the treatment of atherosclerosis. Cell Metab. 7, 365-375 https://doi.org/10.1016/j.cmet.2008.03.001

Temel RE, Brown JM (2012): Biliary and nonbiliary contributions to reverse cholesterol transport. Curr. Opin. Lipidol. 23, 85-90 https://doi.org/10.1097/MOL.0b013e3283508c21

Temel RE, Brown JM (2015): A new model of reverse cholesterol transport: enTICEing strategies to stimulate intestinal cholesterol excretion. Trends Pharmacol. Sci. 36, 440-451 https://doi.org/10.1016/j.tips.2015.04.002

van der Velde AE, Vrins CL, van den Oever K, Kunne C, Oude Elferink RP, Kuipers F, Groen AK (2007): Direct intestinal cholesterol secretion contributes significantly to total fecal neutral sterol excretion in mice. Gastroenterology 133, 967-975 https://doi.org/10.1053/j.gastro.2007.06.019

van der Velde AE, Vrins CL, van den Oever K, Seemann I, Oude Elferink RP, van Eck M, Kuipers F, Groen AK (2008): Regulation of direct transintestinal cholesterol excretion in mice. Am. J. Physiol. Gastrointest. Liver Physiol. 295, G203-208 https://doi.org/10.1152/ajpgi.90231.2008

van der Veen JN, van Dijk TH, Vrins CL, van Meer H, Havinga R, Bijsterveld K, Tietge UJ, Groen AK, Kuipers F (2009): Activation of the liver $\mathrm{X}$ receptor stimulates trans-intestinal excretion of plasma cholesterol. J. Biol. Chem. 284, 19211-19219 https://doi.org/10.1074/jbc.M109.014860

Vrins CL, Ottenhoff R, van den Oever K, de Waart DR, Kruyt JK, Zhao Y, Van Berkel TJ, Havekes LM, Aerts JM, Van Eck M (2012): Trans-intestinal cholesterol efflux is not mediated through high density lipoprotein. J. Lipid Res. 53, 2017-2023 https://doi.org/10.1194/jlr.M022194

Wen S, Jadhav KS, Williamson DL, Rideout TC (2013): Treadmill exercise training modulates hepatic cholesterol metabolism and circulating PCSK9 concentration in high-fat-fed mice. J. Lipids 2013, 908048

https://doi.org/10.1155/2013/908048

Wilund KR, Feeney LA, Tomayko EJ, Chung HR, Kim K (2008): Endurance exercise training reduces gallstone development in mice. J. Appl. Physiol. 104, 761-765 https://doi.org/10.1152/japplphysiol.01292.2007

Received: May 8, 2017

Final version accepted: October 4, 2017 


\title{
Exercise training increased gene expression of LDL-R and PCSK9 in intestine: link to transintestinal cholesterol excretion
}

\author{
Zahra Farahnak ${ }^{1}$, Natalie Chapados ${ }^{2,3}$ and Jean-Marc Lavoie ${ }^{1}$ \\ 1 Department of Kinesiology, University of Montreal, Montreal, QC, Canada \\ 2 Research Institute of Montfort Hospital, Montfort Institute of Knowledge, ON, Canada \\ 3 School of Human Kinetics, Faculty of Health Sciences, University of Ottawa, Ottawa, ON, Canada
}

Table S1. Diet description

\begin{tabular}{lcc}
\hline & SD & SD+Chol (0.25\%) \\
& $(\mathrm{D} 12450 \mathrm{~J})$ & $(\mathrm{D} 13020701)$ \\
\hline Protein (\%) & 19.2 & 19.2 \\
Carbohydrate (\%) & 67.3 & 67.1 \\
Fat (\%) & 4.3 & 4.3 \\
\hline Casein (g) & 200 & 200 \\
L-Cystine (g) & 3 & 3 \\
Corn Starch (g) & 506.2 & 506.2 \\
Maltodextrin 10 (g) & 125 & 125 \\
Sucrose (g) & 68.8 & 68.8 \\
Cellulose, BW200 (g) & 50 & 50 \\
Soybean Oil (g) & 25 & 25 \\
Lard (g) & 20 & 20 \\
Mineral Mix S10026 (g) & 10 & 10 \\
DiCalcium Phosphate (g) & 13 & 13 \\
Calcium Carbonate(g) & 5.5 & 5.5 \\
Potassium Citrate, 1 H2O (g) & 16.5 & 16.5 \\
Vitamin Mix V10001(g) & 10 & 10 \\
Choline Bitartrate (g) & 2 & 2 \\
Cholesterol (g) & 0.0 & 2.63 \\
\hline Kcal/g & 3.85 & 3.84 \\
\hline
\end{tabular}

SD, standard diet; Chol, cholesterol. Formulated by: Research Diets, Inc. (20 Jules Lane, New Brunswick, NJ 08901 USA).
Table S2. Oligonucleotide primers used for quantitative real-time polymerase chain reaction

\begin{tabular}{lll}
\hline Gene & Oligo FWD & Oligo REV \\
\hline$A B C B 1 a$ & ccaccagttcatcgactcac & gatgtgaggctgtctgacga \\
$A B C B 1 b$ & cacagaccgtcagcgaca & caatgcccgtgtaatagtaggc \\
$A B C G 5$ & cggagagttggtgttctgtg & caccgatgtcaagtccatgt \\
$A B C G 8$ & cagatgctggctatcataggg & ctgatttcatcttgccacca \\
LDL- $R$ & tgctactggccaaggacat & ctgggtggtcggtacagtg \\
$P C S K 9$ & cacctagcaggtgtggtcag & gcagactgtgcagactggtg \\
SR-B1 & ggtgcccatcatttaccaac & gcgagccctttttactacca \\
$S R E B P-2$ & gtgcagacagtcgctacacc & aatctgaggctgaaccagga \\
$A c t b$ & cccgcgagtacaaccttct & cgtcatccatggcgaact \\
GAPDH & ccctcaagattgtcagcaatg & agttgtcatggatgaccttgg \\
\hline
\end{tabular}



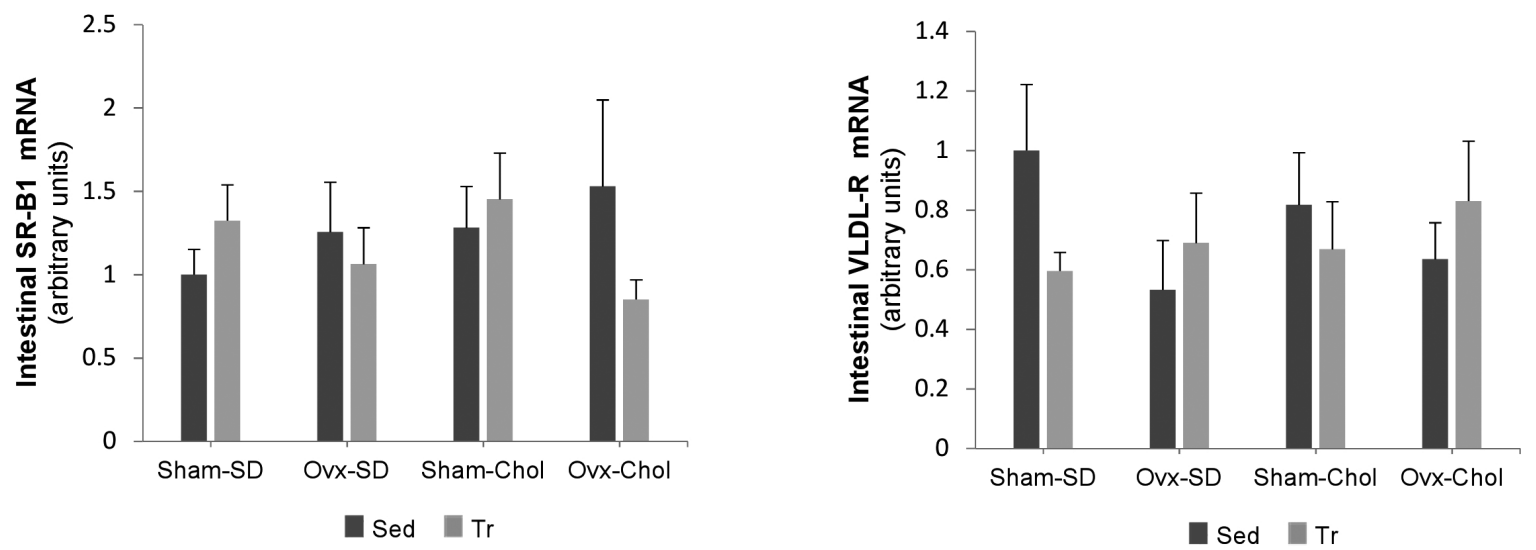

Figure S1. mRNA expression of genes involved in transintestinal cholesterol excretion (TICE) at the basolateral membrane of the intestine in sham operated rats fed a standard diet (Sham-SD), ovariectomized rats fed a standard diet (Ovx-SD), Sham rats fed a standard diet $+0.25 \%$ cholesterol (Sham-Chol) and Ovx rats fed the Chol diet (Ovx-Chol) in sedentary (Sed) or trained (Tr) state. Values are mean \pm SE. SR-B1, scavenger receptor class B member 1; VLDL-R, very-low-density-lipoprotein receptor. 